\title{
Quasi-exactly solvable models in nonlinear optics
}

\author{
G Álvarez, F Finkel, A González-López and M A Rodríguez \\ Departamento de Física Teórica II, Facultad de Ciencias Físicas, Universidad \\ Complutense, 28040 Madrid, Spain
}

\begin{abstract}
We study a large class of models with an arbitrary (finite) number of degrees of freedom, described by Hamiltonians which are polynomial in bosonic creation and annihilation operators, and including as particular cases $n$-th harmonic generation and photon cascades. For each model, we construct a complete set of commuting integrals of motion of the Hamiltonian, fully characterize the common eigenspaces of the integrals of motion, and show that the action of the Hamiltonian in these common eigenspaces can be represented by a quasi-exactly solvable reduced Hamiltonian, whose expression in terms of the usual generators of $\mathfrak{s l}_{2}$ is computed explicitly.

PACS numbers: 03.65.Fd, 42.65Ky
\end{abstract}

Submitted to: J. Phys. A: Math. Gen. 


\section{Introduction}

Dating back at least to 1990, when Zaslavskii [1] pointed out the relation between some quasi-exactly solvable (QES) systems [2, 3, , , 5, 6, 7] and the Dicke and Heisenberg models, there has been an ongoing interest in the correspondence between the exactly calculable part of the spectrum of QES systems and some finite-dimensional systems like the spin systems discussed by Zaslavskii or the effective Hamiltonians frequently used in nonlinear optics.

In essence, Zaslavskii's method consisted in making a suitable ansatz for the coefficients of the eigenvectors of each "multiplet" of the spin system, coefficients which could be grouped into a generating function that turned out to be related by elementary transformations to the wavefunction of the QES system.

In 1995, Álvarez and Álvarez-Estrada [8] studied the usual second harmonic generation effective Hamiltonian by showing its equivalence to an infinite family of onedimensional QES systems. In their method, the transition from the "discrete" photon system to the "continuous" QES systems is achieved by first transforming the effective Hamiltonian from the second quantization to the Bargmann representation. The key idea to separate variables and identify the one-dimensional QES systems - the second step of their procedure - is to use as a variable an appropriate quotient of powers of the Bargmann variables that describe each oscillator. (Later, the same authors [9] used this method to study the problem of third harmonic generation and pointed out that it works in general for the $n$-th harmonic generation.)

Two recent papers by Dolya and Zaslavskii [10, 11] proceed in a related but different direction: they study models with a single degree of freedom whose Hamiltonians are even polynomials in the creation and annihilation operators, and show that under certain conditions these Hamiltonians also lead to a QES system.

Finally, an important idea for our work can be traced back to the early papers on QES models by Ushveridze and Zaslavskii, and appears with more or less emphasis in [1, 8, 6, 11, 9]: the role played by integrals of motion to reduce completely integrable Hamiltonians with more than one degree of freedom to a family of QES systems.

In this paper we study a large class of models with an arbitrary (finite) number of degrees of freedom described by Hamiltonians which are polynomial in the creation and annihilation operators. These models include as particular cases the effective Hamiltonians of $n$-th harmonic generation [12, 13, as well as photon cascades of equal or different frequencies [14], which, to the best of the authors' knowledge, have not been analyzed before as QES systems. For each model, we first construct a complete set of commuting integrals of motion of the Hamiltonian. We use the Bargmann representation to completely characterize the common eigenspaces of the integrals of motion, which are of course invariant under the Hamiltonian. We show that the action

of the Hamiltonian in these common eigenspaces can be represented by a QES reduced Hamiltonian, whose expression in terms of the usual generators of $\mathfrak{s l}_{2}$ is computed explicitly. We emphasize that the derivation of this explicit expression is not just an 
exercise of academic interest but the starting point for the application of asymptotic methods to calculate the corresponding eigenvalues in the limit of a large number of photons. By way of example, we give explicitly the expressions for $n$-th harmonic generation and $N$-photon cascades, which in the former case can be compared with the particular instances $n=2,3$ studied in [8, 9]. The paper ends with a brief summary.

\section{The models}

In this paper we shall study the following general Hamiltonian

$$
H=H_{0}+g H_{1}
$$

where

$$
\begin{array}{lll}
H_{0}=\sum_{l=1}^{N} \nu_{l} a_{l}^{\dagger} a_{l}+\sum_{k=1}^{M} \mu_{k} b_{k}^{\dagger} b_{k} \quad \nu_{l}, \mu_{k}>0 & N, M \in \mathbb{N} \\
H_{1}=\prod_{k=1}^{M}\left(b_{k}^{\dagger}\right)^{m_{k}} \cdot \prod_{l=1}^{N} a_{l}^{n_{l}}+\prod_{l=1}^{N}\left(a_{l}^{\dagger}\right)^{n_{l}} \cdot \prod_{k=1}^{M} b_{k}^{m_{k}} & n_{l}, m_{k} \in \mathbb{N}
\end{array}
$$

and the frequencies $\nu_{l}$ and $\mu_{k}$ are subject to the energy-conservation constraint

$$
\sum_{l=1}^{N} n_{l} \nu_{l}=\sum_{k=1}^{M} m_{k} \mu_{k} .
$$

In the preceding expressions, the operators $a_{l}, b_{k}\left(a_{l}^{\dagger}, b_{k}^{\dagger}\right)$ represent boson annihilation (creation) operators with frequencies $\nu_{l}, \mu_{k}$, respectively. The Hamiltonian (1) is an effective model describing the conversion of a number of photons of frequencies $\nu_{1}, \ldots, \nu_{N}$ into photons of frequencies $\mu_{1}, \ldots, \mu_{M}$. It includes as particular cases the usual processes of $n$-th harmonic generation (if $N=M=m_{1}=1, n_{1}=n$ ) and multiple photon cascades (if $M=m_{1}=n_{1}=\cdots=n_{N}=1$ ), which have recently received considerable attention in the literature [12, 13, 14].

A key property common to all these models is that they are completely integrable in the sense that there exists a set of $N+M$ (the number of degrees of freedom) pairwise commuting, functionally independent operators including the Hamiltonian. These sets are clearly not unique. We have found it convenient to take as our set

$$
\left\{H_{0}, H_{1}, A_{l}, B_{k} \mid l=1, \ldots, N-1 ; k=1, \ldots, M-1\right\}
$$

which includes the unperturbed Hamiltonian $H_{0}$ and the perturbation $H_{1}$ separately, and where

$$
\begin{aligned}
& A_{l}=n_{l+1} a_{l}^{\dagger} a_{l}-n_{l} a_{l+1}^{\dagger} a_{l+1} \\
& B_{k}=m_{k+1} b_{k}^{\dagger} b_{k}-m_{k} b_{k+1}^{\dagger} b_{k+1} .
\end{aligned}
$$

A straightforward computation using the standard bosonic commutation relations shows that these operators commute pairwise, and we shall see below that they are in fact functionally independent. As a consequence, there exists a basis of common eigenfunctions of all the operators in the set. We will see in the next section that 
it is possible to give an explicit description of the simultaneous eigenspaces of $H_{0}, A_{l}$ and $B_{k}$ (the "unperturbed eigenspaces"). By studying the action of $H_{1}$ within these subspaces, in the next section we shall establish the exact solvability of the model (1) -(4) and derive some general properties of its spectrum.

\section{Invariant subspaces and matrix representation}

Following [8], we shall study the spectrum of the Hamiltonian (11) using the Bargmann representation [15]. In this representation, the Hilbert space for a single boson is the space of entire functions of the form

$$
f(z)=\sum_{n=0}^{\infty} \frac{c_{n}}{\sqrt{n !}} z^{n} \quad z \in \mathbb{C}
$$

where the complex numbers $c_{n}$ are such that

$$
\sum_{n=0}^{\infty}\left|c_{n}\right|^{2}<\infty
$$

and the scalar product is defined by

$$
(g, f)=\frac{1}{\pi} \int_{\mathbb{R} \times \mathbb{R}} \mathrm{d}(\operatorname{Re} z) \mathrm{d}(\operatorname{Im} z) \overline{g(z)} f(z) \mathrm{e}^{-|z|^{2}} .
$$

The orthonormal harmonic oscillator eigenstates $|n\rangle$ are given in this representation by

$$
|n\rangle \rightarrow \frac{z^{n}}{\sqrt{n !}} \quad n=0,1, \ldots
$$

and the annihilation and creation operators are represented by derivation with respect to $z$ and multiplication by $z$, respectively

$$
a \rightarrow \frac{\mathrm{d}}{\mathrm{d} z} \quad a^{\dagger} \rightarrow z
$$

The Hilbert space $\mathcal{H}$ of the model (11) is the tensor product of $N+M$ single boson Hilbert spaces. Denoting by $x_{l}(l=1, \ldots, N)$ and $y_{k}(k=1, \ldots, M)$ the complex variables associated to the " $a$ " and " $b$ " degrees of freedom, respectively, we have the following assignments:

$$
a_{l} \rightarrow \partial_{x_{l}} \quad a_{l}^{\dagger} \rightarrow x_{l} \quad b_{k} \rightarrow \partial_{y_{k}} \quad b_{k}^{\dagger} \rightarrow y_{k} .
$$

A basis of the Hilbert space $\mathcal{H}$ of our models is the set of (in general unnormalized) monomials

$$
x^{i} y^{j} \quad i \in \mathbb{Z}_{+}^{N}, j \in \mathbb{Z}_{+}^{M}
$$

where $\mathbb{Z}_{+}$denotes the set of nonnegative integers, and we have used the multiindex notation

$$
x^{i} \equiv \prod_{l=1}^{N} x_{l}^{i_{l}} \quad y^{j} \equiv \prod_{k=1}^{M} y_{k}^{j_{k}} .
$$


The corresponding expressions for the operators in the set (5) are

$$
\begin{aligned}
& H_{0}=\sum_{l=1}^{N} \nu_{l} x_{l} \partial_{x_{l}}+\sum_{k=1}^{M} \mu_{k} y_{k} \partial_{y_{k}} \\
& H_{1}=y^{m} \partial_{x}^{n}+x^{n} \partial_{y}^{m} \\
& A_{l}=n_{l+1} x_{l} \partial_{x_{l}}-n_{l} x_{l+1} \partial_{x_{l+1}} \\
& B_{k}=m_{k+1} y_{k} \partial_{y_{k}}-m_{k} y_{k+1} \partial_{y_{k+1}}
\end{aligned}
$$

where $x^{n}$ and $y^{m}$ are monomials as in equation (15) and

$$
\partial_{x}^{n} \equiv \prod_{l=1}^{N} \partial_{x_{l}}^{n_{l}} \quad \partial_{y}^{m} \equiv \prod_{k=1}^{M} \partial_{y_{k}}^{m_{k}} .
$$

(In the rest of the paper we shall make frequent use of the multiindex notation without further notice whenever there are no ambiguities in the interpretation of the formulae.)

We first note that each monomial (14) is a common eigenfunction of the operators $H_{0}, A_{l}, B_{k}, l=1, \ldots, N-1, k=1, \ldots, M-1$, with eigenvalues respectively given by

$$
\begin{aligned}
& E_{0}=\sum_{l=1}^{N} \nu_{l} i_{l}+\sum_{k=1}^{M} \mu_{k} j_{k} \\
& \alpha_{l}=n_{l+1} i_{l}-n_{l} i_{l+1} \quad l=1, \ldots, N-1 \\
& \beta_{k}=m_{k+1} j_{k}-m_{k} j_{k+1} \quad k=1, \ldots, M-1 .
\end{aligned}
$$

The spectrum of the Hamiltonian (11) can thus be computed by diagonalizing the perturbation Hamiltonian $H_{1}$ in the common eigenspaces $\mathcal{S}_{E_{0}, \alpha_{1}, \ldots, \alpha_{N-1}, \beta_{1}, \ldots, \beta_{M-1}}$, spanned by the monomials (14) whose exponents satisfy equations (21)-(23) for fixed values of $E_{0}, \alpha_{1}, \ldots, \beta_{M-1}$.

We shall now describe the common eigenspaces $\mathcal{S}_{E_{0}, \alpha_{1}, \ldots, \alpha_{N-1}, \beta_{1}, \ldots, \beta_{M-1}} \equiv \mathcal{S}$. Since the unperturbed energy $E_{0}$ is a finite sum of nonnegative terms, it follows that $\mathcal{S}$ is finite-dimensional. Thus, the model (1) is exactly solvable, since its whole spectrum can be computed algebraically.

Let $x^{p} y^{q}$ be a given (fixed) monomial in $\mathcal{S}$. We shall first prove that $\mathcal{S}$ is spanned by monomials of the form

$$
f_{s}=x^{p} y^{q} \zeta^{s}
$$

where

$$
\zeta=\frac{x^{n}}{y^{m}}
$$

and $s$ ranges over a finite interval $s_{0} \leq s \leq s_{1}$ of $\mathbb{Z}$.

Indeed, let $x^{p^{\prime}} y^{q^{\prime}}$ be any other monomial in $\mathcal{S}$. The quotient $Q=x^{p^{\prime}-p} y^{q^{\prime}-q}$ is then a generalized monomial (i.e. a monomial with possibly negative integer exponents) and thus may not belong to $\mathcal{H}$. However, the action of the operators (16)-(19) on such generalized monomials is well-defined, and a simple calculation shows that $Q$ satisfies the system of first-order partial differential equations

$$
H_{0} Q=A_{1} Q=\cdots=A_{N-1} Q=B_{1} Q=\cdots=B_{M-1} Q=0 .
$$


In other words, $Q$ must be a joint invariant of the $M+N-1$ commuting vector fields $H_{0}, A_{l}, B_{k}$. A second straightforward computation shows that the function $\zeta$ defined in (25) is a joint invariant of this set of vector fields. Since the number of independent variables is $N+M$, the general solution of the system (26) is an arbitrary (smooth) function of $\zeta$, and since $Q$ is a generalized monomial, it must be a power of $\zeta$, which proves the first part of our claim.

Since $\mathcal{S}$ is finite-dimensional, the values of $s$ for which $f_{s}$ lies in $\mathcal{S}$ must be bounded above and below. If

$$
s_{0}=\min \left\{s \in \mathbb{Z}: f_{s} \in \mathcal{S}\right\} \quad s_{1}=\max \left\{s \in \mathbb{Z}: f_{s} \in \mathcal{S}\right\}
$$

we shall next prove that

$$
\mathcal{S}=\left\langle f_{s} \mid s_{0} \leq s \leq s_{1}\right\rangle
$$

where \langle\rangle denotes the linear span. Indeed, since clearly

$$
H_{0} f_{s}=E_{0} f_{s} \quad A_{l} f_{s}=\alpha_{l} f_{s} \quad B_{k} f_{s}=\beta_{k} f_{s}
$$

for all integer values of $s$, it suffices to verify that for all $s \in\left[s_{0}, s_{1}\right] \cap \mathbb{Z}$ the exponents of $f_{s}$ are all nonnegative integers. Since $s \in\left[s_{0}, s_{1}\right]$ and both $f_{s_{0}}$ and $f_{s_{1}}$ belong to $\mathcal{S}$, this follows from the inequalities

$$
p_{l}+n_{l} s \geq p_{l}+n_{l} s_{0} \geq 0 \quad q_{k}-m_{k} s \geq q_{k}-m_{k} s_{1} \geq 0 .
$$

This completes the proof of our claim.

Note that (27) and the inequalities (30) imply that the bounds $s_{0}$ and $s_{1}$ are given by

$s_{0}=\max \left\{-\left[\frac{p_{l}}{n_{l}}\right]: 1 \leq l \leq N\right\} \quad s_{1}=\min \left\{\left[\frac{q_{k}}{m_{k}}\right]: 1 \leq k \leq M\right\}$

where [.] denotes the integer part. Calling

$$
\mathcal{N}_{l}=p_{l}+s_{0} n_{l} \geq 0 \quad \mathcal{M}_{k}=q_{k}-s_{0} m_{k} \geq 0
$$

the eigenspace $\mathcal{S}$ can be alternatively written as

$$
\mathcal{S} \equiv \mathcal{S}_{\mathcal{M}}^{\mathcal{N}}=x^{\mathcal{N}} y^{\mathcal{M}}\left\langle 1, \zeta, \ldots, \zeta^{r}\right\rangle
$$

where

$$
r=\min \left\{\left[\frac{\mathcal{M}_{k}}{m_{k}}\right]: 1 \leq k \leq M\right\}
$$

and the nonnegative integers $\mathcal{N}_{l}$ and $\mathcal{M}_{k}$ are subject to the single restriction

$$
\mathcal{N}_{l}<n_{l} \quad \text { for al least one } l \in\{1, \ldots, N\}
$$

which is an immediate consequence of the definition of $s_{0}$. The set of $N+M$ nonnegative integers $\left\{\mathcal{N}_{1}, \ldots, \mathcal{N}_{N}, \mathcal{M}_{1}, \ldots, \mathcal{M}_{M}\right\}$ subject to the condition (35) define uniquely the eigenspace $\mathcal{S}$. Indeed, $\mathcal{N}_{l}$ is the minimum power of $x_{l}$ and $\mathcal{M}_{k}$ is the maximum power of $y_{k}$ of the monomials in $\mathcal{S}$. We have thus proved the main result of this section:

Theorem 1 The common eigenspaces of the operators $H_{0}, A_{l}, B_{k}, l=1, \ldots, N-1$, $k=1, \ldots, M-1$, are the spaces $\mathcal{S}_{\mathcal{M}}^{\mathcal{N}}$ given in equations (33)-(35). 
Since these eigenspaces $\mathcal{S}_{\mathcal{M}}^{\mathcal{N}}$ are invariant under $H_{1}$ (because this operator commutes with $H_{0}$ and $A_{l}, B_{k}$ for all $\left.l, k\right)$, we devote the next paragraphs to study the corresponding action.

Consider the basis $\mathcal{B}$ of $\mathcal{S}_{\mathcal{M}}^{\mathcal{N}}$ spanned by the normalized vectors

$$
e_{s}=\frac{1}{\sqrt{C_{s}}} x^{\mathcal{N}+s n} y^{\mathcal{M}-s m} \quad s=0,1, \ldots, r
$$

where (see equation (11))

$$
C_{s}=\prod_{l=1}^{N}\left(\mathcal{N}_{l}+n_{l} s\right) ! \cdot \prod_{k=1}^{M}\left(\mathcal{M}_{k}-m_{k} s\right) !
$$

A straightforward computation using equations (17), (36) and (37) yields

$$
\begin{aligned}
H_{1} e_{s}=\sqrt{\frac{C_{s-1}}{C_{s}}} \prod_{l=1}^{N}\left(\mathcal{N}_{l}+n_{l} s\right) \cdots\left(\mathcal{N}_{l}+n_{l}(s-1)+1\right) e_{s-1} \\
\quad+\sqrt{\frac{C_{s+1}}{C_{s}}} \prod_{k=1}^{M}\left(\mathcal{M}_{k}-m_{k} s\right) \cdots\left(\mathcal{M}_{k}-m_{k}(s+1)+1\right) e_{s+1} .
\end{aligned}
$$

Thus, the matrix $\mathrm{H}_{1}$ representing $H_{1}$ in the basis $\mathcal{B}$ is tridiagonal, Hermitian, and has zero diagonal entries. The only nonzero entries of $\mathrm{H}_{1}$ are given by

$$
\left(\mathrm{H}_{1}\right)_{s+1, s}=\left(\prod_{l=1}^{N} \prod_{j_{l}=0}^{n_{l}-1}\left(\mathcal{N}_{l}+n_{l} s+j_{l}+1\right) \cdot \prod_{k=1}^{M} \prod_{i_{k}=0}^{m_{k}-1}\left(\mathcal{M}_{k}-m_{k} s-i_{k}\right)\right)^{\frac{1}{2}}=\left(\mathrm{H}_{1}\right)_{s, s+1}
$$

with $s=0,1, \ldots, r-1$.

A number of immediate conclusions can be drawn from the structure of the matrix $\mathrm{H}_{1}$. We note in the first place that $H_{1}$ acts irreducibly in $\mathcal{S}_{\mathcal{M}}^{\mathcal{N}}$. This implies that $H_{1}$ is functionally independent from $H_{0}, A_{l}$ and $B_{k}$ for all $k, l$. Since $x^{n} y^{m}$ is annihilated by all the $A_{l}$ and $B_{k}$ but not by $H_{0}$, the latter operator is functionally independent from the former ones. Since all the $A_{l}$ and $B_{k}$ are clearly functionally independent, the preceding arguments show the functional independence of all the operators in the set (5) and finish the proof of the complete integrability claimed at the end of section 2 .

Secondly, the eigenvalues of $\mathrm{H}_{1}$ are real, simple, and symmetrically distributed around zero. Indeed, let $\delta_{s}(E), 1 \leq s \leq r+1$, be the $s$-th principal minor of the matrix $E \mathbb{I}-\mathrm{H}_{1}$. A straightforward computation shows that $\delta_{s}(E)$ satisfies the three-term recursion relation

$$
\delta_{s+1}(E)=E \delta_{s}(E)-h_{s}^{2} \delta_{s-1}(E) \quad s \geq 1
$$

where $h_{s}=\left(\mathrm{H}_{1}\right)_{s, s-1}$ and $\delta_{1}(E)=E, \delta_{0}(E)=1$. Since $h_{s}^{2}>0$ for $1 \leq s \leq r$, lemma 1 (section 1.8) of [16] implies that the roots of the characteristic polynomial $\delta_{r+1}(E)$ are real and simple. They are also symmetrically distributed around zero, for $\delta_{r+1}(E)$ contains only either even or odd powers of $E$ on account of the form of the recursion relation (40). 
Remark. The model (11) admits certain solvable (and in some cases integrable) generalizations. In the first place, we can replace $g H_{1}$ by any polynomial in $H_{1}$ with real coefficients. Clearly, the resulting Hamiltonian is completely integrable, since it commutes with the operators (5). Moreover, this generalized Hamiltonian preserves the finite-dimensional subspaces $\mathcal{S}_{\mathcal{M}}^{\mathcal{N}}$ and is therefore exactly solvable.

More generally, one can consider a perturbation Hamiltonian $H_{1}^{\prime}$ consisting of a finite sum of terms of the form (3), provided that the exponents in each term satisfy condition (4). In other words,

$$
H_{1}^{\prime}=\sum_{(n, m) \in S} g_{n m}\left(\prod_{k=1}^{M}\left(b_{k}^{\dagger}\right)^{m_{k}} \cdot \prod_{l=1}^{N} a_{l}^{n_{l}}+\prod_{l=1}^{N}\left(a_{l}^{\dagger}\right)^{n_{l}} \cdot \prod_{k=1}^{M} b_{k}^{m_{k}}\right)
$$

where $S$ is any finite subset of the set of all pairs $(n, m) \in \mathbb{Z}_{+}^{N} \times \mathbb{Z}_{+}^{M}$ satisfying the condition (幽). Note that $H_{0}$ still commutes with $H_{1}^{\prime}$, since it commutes separately with each term in the sum (41). Thus the spectrum of $H$ can be computed by diagonalizing the new perturbation Hamiltonian $H_{1}^{\prime}$ in the eigenspaces of $H_{0}$, which are finite-dimensional by equation (21). Hence this more general model is also exactly solvable. Note, however, that now to each term in the sum (41) there correspond two families of operators $A_{l} \equiv A_{l}(n)$ and $B_{k} \equiv B_{k}(m)$ which do not necessarily commute with the remaining terms in $H_{1}^{\prime}$. Hence, this model need not be completely integrable for general values of the coupling constants $g_{n m}$.

\section{Quasi-exact solvability of the reduced Hamiltonians}

In the previous section we have shown that the computation of the spectrum of the Hamiltonian (1) is equivalent to the diagonalization of a family of tridiagonal Hermitian matrices with zero diagonal elements. For small values of $r+1=\operatorname{dim} \mathcal{S}_{\mathcal{M}}^{\mathcal{N}}$, the eigenvalues of the matrix $\mathrm{H}_{1}$ representing $H_{1}$ can be easily computed in closed form. In many situations of physical interest, however, the number of photons of each frequency involved can actually be very large. This implies (see equation (34)) that the parameter $r$ can also take large values. Although the spectrum of $\mathrm{H}_{1}$ can be determined numerically for fixed values of the parameters $\mathcal{N}_{l}, \mathcal{M}_{k}$ labelling the space $\mathcal{S}_{\mathcal{M}}^{\mathcal{N}}$, one is often interested in the behavior of its eigenvalues as a function of $\mathcal{N}_{l}, \mathcal{M}_{k}$ (and thus $r$ ). In the case of second-harmonic generation, i.e. $N=M=m_{1}=1, n_{1}=2$, Álvarez and ÁlvarezEstrada 88 derived asymptotic formulae for the eigenvalues of $\mathrm{H}_{1}$ by performing a semiclassical analysis of the second-order ordinary differential equation (ODE) obtained by restricting the perturbation Hamiltonian $H_{1}$ to $\mathcal{S}_{\mathcal{M}_{1}}^{\mathcal{N}_{1}}=\mathcal{S}_{r}^{\epsilon}$, where $\epsilon=0,1$. It was also shown in that paper that the restriction of $H_{1}$ to $\mathcal{S}_{r}^{\epsilon}$ is in fact equivalent to a QES operator in a single variable, in agreement with previous results due to Zaslavskii [1].

In this section we show that the restriction of our general perturbation $H_{1}$ to an eigenspace $\mathcal{S}_{\mathcal{M}}^{\mathcal{N}}$ is also equivalent to a QES differential operator in a single variable. Besides the purely mathematical interest of this result, the knowledge of explicit expressions for the ordinary differential operator of the restriction $\left.H_{1}\right|_{\mathcal{S}_{\mathcal{M}}^{\mathcal{M}}}$ is precisely 
the starting point to apply asymptotic techniques, which are currently being developed for third- and higher-order ODE's.

Consider the action of $H_{1}$ on an element $x^{\mathcal{N}} y^{\mathcal{M}} P(\zeta) \in \mathcal{S}_{\mathcal{M}}^{\mathcal{N}}$

$H_{1}\left(x^{\mathcal{N}} y^{\mathcal{M}} P(\zeta)\right)=\prod_{k=1}^{M} y_{k}^{m_{k}+\mathcal{M}_{k}} \cdot \prod_{l=1}^{N} \partial_{x_{l}}^{n_{l}}\left(x_{l}^{\mathcal{N}_{l}} P(\zeta)\right)+\prod_{l=1}^{N} x_{l}^{n_{l}+\mathcal{N}_{l}} \cdot \prod_{k=1}^{M} \partial_{y_{k}}^{m_{k}}\left(y_{k}^{\mathcal{M}_{k}} P(\zeta)\right)$

where $P$ is a polynomial of degree at most $r$. Since

$$
\begin{aligned}
& \partial_{x_{l}}^{n_{l}}\left(x_{l}^{\mathcal{N}_{l}} P(\zeta)\right)=x_{l}^{\mathcal{N}_{l}-n_{l}} \prod_{j_{l}=0}^{n_{l}-1}\left(\mathcal{N}_{l}-j_{l}+n_{l} \zeta \partial_{\zeta}\right) P(\zeta) \\
& \partial_{y_{k}}^{m_{k}}\left(y_{k}^{\mathcal{M}_{k}} P(\zeta)\right)=y_{k}^{\mathcal{M}_{k}-m_{k}} \prod_{i_{k}=0}^{m_{k}-1}\left(\mathcal{M}_{k}-i_{k}-m_{k} \zeta \partial_{\zeta}\right) P(\zeta)
\end{aligned}
$$

it follows that

$$
H_{1}\left(x^{\mathcal{N}} y^{\mathcal{M}} P(\zeta)\right)=x^{\mathcal{N}} y^{\mathcal{M}} H_{1, \text { red }} P(\zeta)
$$

where the reduced Hamiltonian is given by

$$
H_{1, \mathrm{red}}=\frac{1}{\zeta} \prod_{l=1}^{N} \prod_{j_{l}=0}^{n_{l}-1}\left(\mathcal{N}_{l}-j_{l}+n_{l} \zeta \partial_{\zeta}\right)+\zeta \prod_{k=1}^{M} \prod_{i_{k}=0}^{m_{k}-1}\left(\mathcal{M}_{k}-i_{k}-m_{k} \zeta \partial_{\zeta}\right)
$$

Thus the action of $\left.H_{1}\right|_{\mathcal{S}_{\mathcal{M}}^{\mathcal{N}}}$ is equivalent to that of $H_{1 \text {,red }}$ on the space $\mathcal{P}_{r}$ of polynomials in $\zeta$ of degree at most $r$. Note in particular that the invariance of $\mathcal{S}_{\mathcal{M}}^{\mathcal{N}}$ under $H_{1}$ implies that $\mathcal{P}_{r}$ is invariant under $H_{1 \text {,red }}$ (this fact can be also verified directly from equation (46) using (34) and (35); see the discussion below).

Let $d=\max \left(\sum_{l=1}^{N} n_{l}, \sum_{k=1}^{M} m_{k}\right)$ be the order of the differential operator $H_{1, \mathrm{red}}$. If $d \leq r$, a well-known theorem due to Turbiner [17] (see [18] for a simplified proof) states that the invariance of the polynomial space $\mathcal{P}_{r}$ under $H_{1 \text {,red }}$ implies that this operator can be written as a polynomial of degree $d$ in the generators of the realization of $\mathfrak{s l}_{2}$ spanned by

$$
J^{+}=\zeta^{2} \partial_{\zeta}-r \zeta \quad J^{0}=\zeta \partial_{\zeta}-\frac{r}{2} \quad J^{-}=\partial_{\zeta}
$$

If $d>r$, Turbiner's theorem only guarantees that the $r$-th order part of $H_{1, \text { red }}$ is a polynomial of degree $d$ in the operators (47). In fact, even if $d>r$ the reduced Hamiltonian $H_{1, \text { red }}$ can be written as a polynomial of degree $d$ in the generators (47). Indeed, choose $k^{\prime} \in\{1, \ldots, M\}$ and $l^{\prime} \in\{1, \ldots, N\}$ such that (see equations (34) and (35))

$$
r=\left[\frac{\mathcal{M}_{k^{\prime}}}{m_{k^{\prime}}}\right] \quad \text { and } \quad \mathcal{N}_{l^{\prime}}<n_{l^{\prime}} .
$$

Since all the factors in the products of the expression (46) commute, it follows that

$$
H_{1, \text { red }}=n^{n} J^{-} \cdot \prod_{j_{l^{\prime}}=0, j_{l^{\prime}} \neq \mathcal{N}_{l^{\prime}}}^{n_{l^{\prime}}-1}\left(J^{0}+\frac{\mathcal{N}_{l^{\prime}}-j_{l^{\prime}}}{n_{l^{\prime}}}+\frac{r}{2}\right) \cdot \prod_{l=1, l \neq l^{\prime}}^{N} \prod_{j_{l}=0}^{n_{l}-1}\left(J^{0}+\frac{\mathcal{N}_{l}-j_{l}}{n_{l}}+\frac{r}{2}\right)
$$




$$
\begin{gathered}
+(-m)^{m} J^{+} \cdot \prod_{i_{k^{\prime}}=0, i_{k^{\prime}} \neq \mathcal{M}_{k^{\prime}} \bmod m_{k^{\prime}}}^{m_{k^{\prime}}-1}\left(J^{0}+\frac{i_{k^{\prime}}-\mathcal{M}_{k^{\prime}}}{m_{k^{\prime}}}+\frac{r}{2}\right) \\
\cdot \prod_{k=1, k \neq k^{\prime}}^{M} \prod_{i_{k}=0}^{m_{k}-1}\left(J^{0}+\frac{i_{k}-\mathcal{M}_{k}}{m_{k}}+\frac{r}{2}\right) .
\end{gathered}
$$

This general expression of the differential operator $H_{1, \text { red }}$ as a polynomial in the generators of the $\mathfrak{s l}_{2}$ algebra is of course not unique, and can be written in different forms using the commutation relations of the generators (47). We conclude this section by showing explicitly the physically most important particular cases, in which the general expression (49) simplifies considerably.

Example 1 Consider the problem of $n$-th harmonic generation, in which $N=M=$ $m_{1}=1, n_{1}=n$ (and therefore the multiindices reduce to ordinary indices). The eigenspaces of $H_{0}$ are $\mathcal{S}_{r}^{\epsilon}=x^{\epsilon} y^{r} \mathcal{P}(\zeta)$, where $r \in \mathbb{Z}_{+}, \epsilon=0, \ldots, n-1$, and $\zeta=x^{n} / y$. The corresponding expression of $H_{1, \text { red }}$ reads

$$
H_{1, \text { red }}=n^{n} J^{-} \prod_{j=0, j \neq \epsilon}^{n-1}\left(J^{0}+\frac{\epsilon-j}{n}+\frac{r}{2}\right)-J^{+} .
$$

This expression can be easily shown to be equivalent to the expressions obtained in [8] for second harmonic generation $(n=2)$ and in [9] for third-harmonic generation $(n=3)$ and $\epsilon=0$ (incidentally, in 9] the cases $\epsilon=1$ and 2 were not considered).

Example 2 In the case of a multiple photon cascade, $M=m_{1}=n_{1}=\cdots=n_{N}=1$, the eigenspaces of $H_{0}$ and $A_{l}, l=1, \ldots, N-1$, are $\mathcal{S}_{r}^{\mathcal{N}}=x^{\mathcal{N}} y^{r} \mathcal{P}(\zeta)$, where $\mathcal{N}_{l^{\prime}}=0$ for some $l^{\prime} \in\{1, \ldots, N\}, r \in \mathbb{Z}_{+}$, and $\zeta=\left(x_{1} \cdots x_{N}\right) / y$. The expression of $H_{1, \text { red }}$ in terms of the generators (47) is

$$
H_{1, \text { red }}=J^{-} \prod_{l=1, l \neq l^{\prime}}^{N}\left(J^{0}+\mathcal{N}_{l}+\frac{r}{2}\right)-J^{+} .
$$

\section{Summary}

In this paper we have established a common framework to deal with a large class of processes in nonlinear optics including the problems of $n$-th harmonic generation and multiple photon cascades. The distinguishing feature of these models from the physical point of view is the condition of energy conservation, which is the key mathematical condition to prove their complete integrability and the exact solvability. By using the Bargmann representation, we have been able to provide an explicit description of the common unperturbed eigenspaces, as well as equally explicit expressions of the action of the perturbation Hamiltonian in these unperturbed eigenspaces. We have also derived some general properties of the spectrum of the restriction of the perturbation $H_{1}$ to each unperturbed eigenspace, such as the nondegeneracy and symmetric distribution of the perturbation energies around zero. The link between these finite matrix representations and the corresponding continuous QES system is most conveniently established by 
the introduction of a new "projective" coordinate, which is a quotient of powers of the Bargmann variables that describe the different oscillators (and physically carries information on the phase difference among the oscillators). Furthermore, we have been able to give explicit expressions of the reduced QES Hamiltonians as polynomials in the generators of the standard QES realization of $\mathfrak{s l}_{2}$ by first-order differential operators.

\section{References}

[1] Zaslavskii O B 1990 Phys. Lett. A 149365

[2] Zaslavskii O B and Ulyanov V V 1984 Zh. Eksp. Teor. Fiz. 871724 (Engl. Transl. 1984 Sov. Phys. JETP 60 991)

[3] Zaslavskii O B and Ulyanov V V 1987 Teor. Mat. Fiz. 71260 (Engl. Transl. 1987 Theor. Math. Phys. 71 520)

[4] Turbiner A V 1988 Commun. Math. Phys. 118467

[5] Shifman M A 1989 Int. J. Mod. Phys. A 42897

[6] Ushveridze A G 1994 Quasi-Exactly Solvable Models in Quantum Mechanics (Bristol: IOP)

[7] González-López A, Kamran N and Olver P J 1994 Contemporary Mathematics 160113

[8] Álvarez G and Álvarez-Estrada R F 1995 J. Phys. A: Math. Gen. 285767

[9] Álvarez G and Álvarez-Estrada R F 2001 J. Phys. A: Math. Gen. 3410045

[10] Dolya S N and Zaslavskii O B 2000 J. Phys. A: Math. Gen. 33 L369

[11] Dolya S N and Zaslavskii O B 2001 J. Phys. A: Math. Gen. 345955

[12] Bajer J and Miranowicz A 2000 J. Opt. B: Quantum Semiclass. Opt. 2 L10

[13] Klimov A B and Sánchez-Soto L L 2000 Phys. Rev. A 61063802

[14] Karassiov V P, Gusev A A and Vinitsky S I 2001 Preprint arXiv quant-ph/0105152

[15] Galindo A and Pascual P 1990 Quantum Mechanics I (Berlin: Springer-Verlag)

[16] Arscott F M 1964 Periodic Differential Equations (Oxford: Pergamon)

[17] Turbiner A 1992 J. Phys. A: Math. Gen. 25 L1087

[18] Finkel F and Kamran N 1998 Adv. Appl. Math. 20300 